\title{
Artefact
}

Techniques, histoire et sciences humaines

\section{Les temporalités d'un grand projet technologique : Énergie atomique du Canada limitée et la trajectoire du réacteur MAPLE-X}

The temporalities of a large technological project: Atomic Energy of Canada

Limited and the trajectory of the reactor MAPLE-X

\section{Mahdi Khelfaoui}

\section{OpenEdition}

Journals

Édition électronique

URL : https://journals.openedition.org/artefact/10495

DOI : 10.4000/artefact.10495

ISSN : 2606-9245

Éditeur :

Association Artefact. Techniques histoire et sciences humaines, Presses universitaires du Midi

\section{Édition imprimée}

Date de publication : 7 octobre 2021

Pagination : 409-436

ISBN : 978-2-8107-0755-3

ISSN : 2273-0753

\section{Référence électronique}

Mahdi Khelfaoui, «Les temporalités d'un grand projet technologique : Énergie atomique du Canada limitée et la trajectoire du réacteur MAPLE-X », Artefact [En ligne], 14 | 2021, mis en ligne le 07 octobre 2021, consulté le 08 octobre 2021. URL : http://journals.openedition.org/artefact/10495 ; DOI : https://doi.org/10.4000/artefact.10495

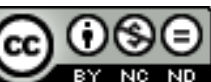

Artefact, Techniques, histoire et sciences humaines est mise à disposition selon les termes de la Licence Creative Commons Attribution - Pas d'Utilisation Commerciale - Pas de Modification 4.0 International. 


\title{
Les temporalités d'un grand projet technologique : Energie atomique du Canada limitée et la trajectoire du réacteur MAPLE-X
}

\author{
Mahdi Khelfaoui
}

\section{Résumé}

À travers l'histoire du réacteur MAPLE-X, un projet nucléaire mené par l'entreprise publique Énergie atomique du Canada limitée, cet article s'intéresse aux temporalités qui rythment les trajectoires des grands projets technologiques (GPT). Longs, complexes, aux enjeux multiples et aux résultats incertains, les GPT sont soumis à différentes contraintes émanant de l'environnement interne et externe des organisations chargées de leur réalisation. Par conséquent, leur déroulement ne se réduit pas à l'écoulement d'un temps linéaire et homogène, mais est plutôt marqué par l'enchevêtrement de différentes temporalités, dont quatre sont identifiées dans le cas du projet MAPLE-X : technique, gestionnaire, réglementaire et politique. C'est dans l'articulation de ces temporalités et les tensions qu'elles exercent les unes sur les autres que se dessinent les trajectoires sinueuses des GPT.

\section{Mots-clés}

Canada, grands projets technologiques, énergie nucléaire, temporalités

95 Mahdi Khelfaoui, «Les temporalités d'un grand projet technologique : Énergie atomique du Canada limitée et la trajectoire du réacteur MAPLE-X », Artefact, 14, 2021, p. 409-436. 


\section{The temporalities of a large. technological project : Atomic Energy of Canada Limited and the trajectory of the reactor MAPLE-X}

\section{Abstract}

Through the history of the MAPLE-X reactor, a nuclear project conducted by the public company Atomic Energy of Canada Limited, this article looks at the temporalities that punctuate the trajectories of Large Technological Projects (LTPs). Long, complex, with multiple stakes and uncertain results, LTPs are subject to various constraints emanating from the internal and external environment of the organizations responsible for their implementation. As a result, their unfolding cannot be reduced to the course of a linear and homogeneous time, since it is rather characterized by the intertwining of different temporalities, four of which are identified in the case of the MAPLE-X project : technical, managerial, regulatory and political. This paper argues that is through the articulation of these temporalities and the tensions they exert on each other that the sinuous trajectories of the LTPs should be analyzed.

\section{Keywords}

Canada, large technological systems, nuclear energy, temporalities

$\mathrm{L}$ 'histoire des grandes infrastructures technologiques, qu'elles soient destinées à la production industrielle, la recherche scientifique, les transports ou l'urbanisation, est souvent abordée sous l'angle de l'histoire des grands projets ${ }^{1}$. De par leur taille, les ressources qu'ils mobilisent et leur caractère innovant, les grands projets technologiques (GPT) se déroulent généralement sur une échelle de plusieurs années, voire de plusieurs décennies. Les organisations chargées de leur réalisation font face

1. Faucher, 1999. 
à des processus incertains et à des événements contingents dont les chances de se produire augmentent à mesure que la durée des projets s'étire dans le temps. Ainsi, le déroulement d'un GPT obéit rarement à une succession logique d'actions coordonnées, même si la prévision et la planification demeurent nécessaires, mais tend plutôt à montrer « un enchevêtrement de dynamiques temporelles, de rationalités techniques, de veto politiques et d'interactions relativement aléatoires ${ }^{2} »$.

Malgré son importance dans le façonnement des trajectoires des GPT, le temps y est souvent abordé comme une variable unidimensionnelle : un temps singulier, "linéaire, régulier et absolu ${ }^{3}$ ». Pourtant, les travaux en histoire et en sociologie des techniques ont depuis longtemps critiqué les représentations linéaires et unidimensionnelles des processus d'innovations sous-tendant les GPT, voulant que ceux-ci obéissent à une succession d'étapes ordonnées procédant de la recherche fondamentale à la mise en production ${ }^{4}$. En effet, ces prétendues étapes non seulement se chevauchent, mais les retours en arrière, au gré de circonstances souvent imprévisibles, sont fréquents ${ }^{5}$. La linéarité des GPT est également mise à mal par l'analyse des prises de décisions qui orientent leurs trajectoires ${ }^{6}$, montrant que celles-ci résultent de situations qui sont " simultanément contingentes et réglées ${ }^{7}$ ». Les prises de décisions dans les GPT constituent non seulement l'aboutissement de longs processus de négociation, à l'intérieur de cadres organisés ou informels, mais sont aussi le fruit de facteurs contingents qui constituent des éléments importants de discontinuité ${ }^{8}$.

À la suite de ces observations, cet article veut montrer, à travers l'histoire $\mathrm{du}$ réacteur canadien MAPLE-X que les trajectoires des GPT résultent de la superposition et de l'enchevêtrement de temporalités multiples. Chacune des temporalités identifiées dans cet article fait intervenir des institutions et des acteurs qui agissent sur le projet MAPLE-X de façon indépendante mais également à travers leurs interactions mutuelles. La notion

\footnotetext{
2. Gilbert Simondon, cité dans Lascoumes et Le Galès, 2007, p. 52.

3. Dubar, 2008.

4. Le « modèle linéaire de l'innovation » a notamment été remis en cause par l'approche constructiviste en sociologie des techniques. Voir Pinch et Bijker, 1984 ; Bijker, Hughes et Pinch (dir.), 1987.

5. Gingras et Khelfaoui, 2013.

6. Pour quelques exemples, voir : Bromberg, 1982 ; Pestre, 1992 ; Velho et Pessoa, 1998 ; McCurdy, 2007.

7. Giraudeau et Graber, 2019.

8. Gingras et Trépanier, 1989.
} 
de temporalités est ici comprise comme "l'inscription dans le temps de différentes séquences d'actions ${ }^{9} »$ marquées par des rythmes et des inerties qui leurs sont propres. Le déroulement des grands projets de recherche scientifique est ainsi structuré, selon les sociologues Philippe Brunet et Michel Dubois, par trois temporalités aux pas de temps différents : une temporalité professionnelle, qui renvoie au travail et à l'activité routinière des chercheurs ; une temporalité gestionnaire, qui impose le cadre temporel et l'organisation du déroulement du projet en jalons successifs ; enfin, une temporalité normative, qui renvoie aux contraintes des cadres légaux pouvant s'exercer sur l'activité scientifique, comme ce qui relève par exemple de l'encadrement éthique de la recherche ${ }^{10}$.

L'analyse du projet MAPLE-X fait ressortir quant à elle quatre temporalités distinctes. Les grands projets de recherches technoscientifiques mobilisant de plus en plus des appareillages complexes qui impliquent "des formes d'organisation industrielle ${ }^{11}$ ", les temporalités proposées pour le projet MAPLE-X reprennent en partie la typologie proposée par Brunet et Dubois, tout en étant adaptées aux caractéristiques des grands projets technologiques, en particulier nucléaires. Ces derniers se distinguent en effet par quatre dimensions : une dimension technique ${ }^{12}$; une dimension réglementaire portant sur la gestion des risques nucléaires (liés par exemple à la sûreté des réacteurs, aux risques environnementaux ou à la gestion des déchets radioactifs) ${ }^{13}$; une dimension gestionnaire qui inclut l'encadrement administratif ainsi que la définition des objectifs stratégiques, économiques et commerciaux des projets $^{14}$; enfin, une dimension politique qui peut non seulement renvoyer aux oppositions sociales soulevées par les grands projets nucléaires, mais également à leur instrumentalisation politique par les gouvernements ${ }^{15}$.

La temporalité technique renvoie au temps de la conception, de l'expérimentation en laboratoire, de la réalisation des études techniques, des

9. Schultz, 2013.

10. Brunet, 2012 ; Brunet et Dubois, 2012.

11. Pestre et Jacq, 1996, p. 167.

12. Pour le cas du Canada, voir Hurst, 1997.

13. Wellock, 2017. Pour le cas français, voir : Mangeon, 2018.

14. Sur les considérations économiques et commerciales des grands projets nucléaires, voir notamment : Cowan, 1990 ; Grubler, 2010.

15. Sur la dimension techno-politique ou socio-politique des grands projets nucléaires français, voir par exemple Hecht, 2014 ; Topçu, 2013 ; Barthe, 2006. 
travaux de chantier ou des tests de mise en service. Dans le cas du projet MAPLE-X elle concerne en premier chef le temps du travail routinier des scientifiques, des ingénieurs et des techniciens au sein de l'entreprise publique Énergie atomique du Canada limitée (ÉACL), maître d'œuvre du projet. Les différentes activités qui définissent la temporalité technique d'un GPT ne s'inscrivent pas nécessairement dans les séquences ordonnées et définies par sa temporalité gestionnaire. Il est ainsi rare que la conception d'un grand projet nucléaire, ou autre, s'arrête là où débute son exécution. Au contraire, les deux étapes demeurent en constante tension et en interaction, le temps de la conception s'apparentant à un processus " ouvert, qui se poursuit dans le chantier jusqu'à la réalisation complète de l'ouvrage ${ }^{16} »$. La temporalité gestionnaire concerne les activités relatives à l'administration du projet et à sa planification séquentielle, l'organisation des échéanciers et des budgets, ainsi qu'à la définition des objectifs stratégiques et commerciaux du projet à l'échelle managériale. Dans plusieurs grands projets nucléaires, qui sont orientés simultanément vers l'exploration technique et l'exploitation commerciale, cette temporalité entre souvent en tension avec la temporalité technique. L'analyse de la trajectoire de technologies nucléaires de pointe, tels que les réacteurs surgénérateurs ou à neutrons rapides, souligne bien l'ambivalence de ces objets " hybrides ${ }^{17}$ " se situant à mi-chemin entre l'appareil de démonstration, dont la trajectoire est soumise à l'incertitude inhérente aux activités de recherche et de développement et qui peut toujours être sujet à modifications, et le produit commercial achevé qui doit être mis sur le marché ou livré à un client à une échéance fixée contractuellement. Le réacteur MAPLE-X n'est pas exempt de cette tension, puisque sa conception est tantôt influencée par des considérations techniques relatives à sa mission de réacteur de recherche, tantôt par des considérations commerciales liées à ses objectifs de production pour le marché mondial des radio-isotopes médicaux.

La temporalité réglementaire renvoie aux démarches de mise en conformité avec les différentes lois et normes industrielles, techniques, environnementales ou sanitaires qui encadrent un GPT. Dans les grands projets nucléaires, la temporalité réglementaire fait généralement intervenir les organismes nationaux de réglementation et de contrôle de la sûreté nucléaire. Dans le cas du projet canadien MAPLE-X, il s'agit de la Commission de contrôle

16. Graber, 2011, p. 17 ; Montel, 1998.

17. Tillemant, Garcias, Minguet et Charue Duboc, 2019 ; Jobert et Le Renard, 2014. 
de l'énergie atomique (CCEA). Si l'influence de la temporalité réglementaire sur le projet MAPLE-X s'exerce à des moments précis du projet, ceux où la CCEA doit officiellement statuer sur la conformité des études techniques sensées garantir la sûreté du réacteur, ses agents entrent également en interaction, ou " en dialogue ${ }^{18}$ ", avec les ingénieurs d'ÉACL pendant la réalisation même des études techniques. La temporalité réglementaire influence donc la temporalité technique de manière ponctuelle mais également dans la continuité du déroulement du projet. Finalement, la temporalité politique, qui fait référence aux contraintes sociales ou politiques qui s'exercent sur un GPT, renvoie à des prises de décisions gouvernementales qui affectent la mission stratégique d'ÉACL et son devenir sur le long terme, au-delà du projet MAPLE-X. Par ailleurs, ces décisions, notamment celles qui touchent à la privatisation partielle d'ÉACL, jouent indirectement le rôle de facteurs contingents qui viennent exercer une influence décisive sur les autres temporalités du projet MAPLE-X.

La suite de cet article est consacrée à la reconstitution des évènements marquants de l'histoire du réacteur MAPLE-X. Ce récit, s'appuyant sur des archives gouvernementales et d'entreprise inédites, s'intéresse d'abord à la genèse du projet au début des années 1980, puis aux différentes contraintes techniques, commerciales, réglementaires et politiques qui pèsent sur sa réalisation et mènent finalement à son abandon en 1993. La conclusion de l'article vient ensuite lier ces évènements aux quatre temporalités définies en introduction et établir explicitement la manière dont celles-ci s'influencent mutuellement pour façonner la trajectoire globale du projet MAPLE-X.

\section{Le réacteur MAPLE-X : la trajectoire d'un grand projet nucléaire}

En 1952, le gouvernement du Canada crée ÉACL pour développer et commercialiser les applications civiles de l'énergie nucléaire ${ }^{19}$. En 1978, l'entreprise compte cinq divisions semi-autonomes, chapeautées par un siège social situé à Toronto, dont les trois plus importantes sont : la Société d'ingénierie, basée à Toronto et responsable du développement et

18. Sur l'approche "dialogique » de la réglementation nucléaire, voir Mangeon et Pallez, 2017.

19. Bothwell, 1988. 
de la commercialisation des centrales électronucléaires canadiennes, dites CANDU ; la Société radiochimique, basée à Ottawa et responsable de la commercialisation de radio-isotopes notamment utilisés en médecine nucléaire ; la Société de la recherche, qui pilote les laboratoires de recherche et développement $(\mathrm{R} \& \mathrm{D})$ de l'entreprise. La Société de la recherche opère trois réacteurs : le NRU et le NRX dans ses laboratoires de Chalk River en Ontario, et le WR-1 sur son site de Whiteshell au Manitoba. La Société de la recherche est étroitement liée aux deux autres divisions de l'entreprise. En plus d'être au cœur des programmes de recherche fondamentale et appliquée, ses réacteurs sont utilisés pour réaliser des travaux de développement en soutien à la Société d'ingénierie, mais aussi pour produire les radio-isotopes médicaux qui sont transférés à la Société radiochimique pour être chimiquement traités et commercialisés.

\section{Genèse et approbation du projet MAPLE-X}

Lidée de concevoir le réacteur MAPLE-X naît au sein de la Société de la recherche au début des années 1980. ÉACL réalise alors une étude de marché pour des petits réacteurs nucléaires de recherche, à la suite de la publication par l'Agence internationale de l'énergie atomique (AIEA) d'un document soulignant le rôle fondamental que pourraient jouer de tels réacteurs dans le développement des programmes nucléaires nationaux. Le document de l'AIEA encourage particulièrement les pays en voie de développement souhaitant initier un programme nucléaire à se doter de réacteurs de recherche permettant une variété d'applications ${ }^{20}$. C'est dans cette perspective que débute le développement du concept MAPLE ${ }^{21}$, dont la première lettre de l'acronyme, " $M$ " pour Multipurpose, indique bien la nature versatile : fournir des informations techniques pertinentes sur une variété de combustibles nucléaires et de matériaux, servir de base expérimentale pour la recherche fondamentale et appliquée, former une main d'œuvre qualifiée et produire des radio-isotopes pour la médecine nucléaire ${ }^{22}$.

En plus de positionner ÉACL sur un potentiel marché de réacteurs de recherche, le développement du concept MAPLE s'inscrit dans un contexte

20. Agence Internationale de l'Énergie Atomique, 1980.

21. MAPLE est un acronyme pour Multipurpose Applied Lattice Experimental.

22. Lidstone, 1984, p. 4. 
des coupures budgétaires subies par l'entreprise avec l'arrivée au gouvernement, en septembre 1984, du Parti progressiste conservateur du Canada. Ce dernier vote dès janvier 1985 une division par deux du budget annuel de R\&D d'ÉACL sur un horizon de cinq ans, souhaitant le faire passer de 200 à 100 millions de dollars canadiens (M\$) entre 1985 et 1990 . En réaction à cette décision, la Société de la recherche d'ÉACL met sur pied un Research Reactor Rationalization Program, qui vise à réorganiser la gestion de ses réacteurs de la façon la plus économique possible, en fonction du nouveau cadre budgétaire établi par le gouvernement ${ }^{23}$. La Société de la recherche détermine que l'ensemble de ses besoins en R\&D pourraient être satisfaits par son réacteur NRU. Seulement, son utilisation à plein potentiel pour la $\mathrm{R} \& \mathrm{D}$ est contrariée par la production des radio-isotopes ${ }^{24}$, activité commerciale qui le mobilise de plus en plus à mesure quaugmentent les besoins de la Société radiochimique dans un marché mondial en pleine croissance.

Le molybdène-99 en particulier, isotope phare de la médecine nucléaire dont la Société radiochimique contrôle $60 \%$ du marché mondial, ne requiert pas un réacteur doté d'une puissance de sortie aussi élevée que celle du NRU ou du NRX, mais exige cependant des flux neutroniques importants. De plus, le molybdène-99 ne peut être stocké, à cause de sa demivie relativement courte de 66 heures, et doit être produit en flux tendu pour être continuellement acheminé vers les compagnies radio-pharmaceutiques clientes de la Société radiochimique. Sa production requiert par conséquent la présence d'un réacteur de réserve, prêt à prendre la relève du réacteur principal, en l'occurrence le NRU, à n'importe quel moment dans l'éventualité où ce dernier tombe en panne ou lorsqu'il est arrêté pour des opérations de maintenance. Le NRX remplit jusqu'à présent cette fonction de réacteur de réserve, mais la Société de la recherche souhaiterait plutôt assigner ce rôle au NRU, afin de lui libérer du temps pour la recherche, et construire un nouveau réacteur principal de production ${ }^{25}$. La construction d'un réacteur de type MAPLE, d'une puissance relativement peu élevée mais en mesure de produire les flux neutroniques requis pour produire des radio-isotopes, permettrait ainsi d'arrêter définitivement les réacteurs

23. Loi canadienne sur l'accès à l'information (LCAI), procès-verbal de la réunion du C.A. d'ÉACL, 25 janvier 1985, p. 17.

24. LCAI, A-2016-00027, «Presentation to the AECL Board of Directors », 25 janvier 1985, p. 2. 25. Ibid. 
NRX et WR-1, dont les activités seraient transférées au NRU. Leur arrêt constituerait par ailleurs une source d'économies non négligeable pour la Société de la recherche.

Afin de minimiser les futurs coûts de construction, le bâtiment du NRX et son blindage seraient réutilisés pour accueillir le MAPLE, dont l'opération servirait dans un premier temps à démontrer son potentiel de réacteur de recherche, avant qu'il ne soit utilisé à long terme pour produire des radio-isotopes médicaux. Une fois l'ensemble des travaux achevés, dans le courant de l'année 1990, la Société de la recherche s'attend à pouvoir économiser $22 \%$ par année sur le budget d'opération de ses réacteurs, soit un montant de $8.4 \mathrm{M} \$$ sur un budget total de $35.7 \mathrm{M} \${ }^{26}$. En somme, la construction d'un réacteur de type MAPLE devient un élément central du programme de rationalisation de la Société de la recherche, puisqu'elle lui permet de trouver à coût réduit un équilibre entre ses activités de $R \& D$ et de production de radio-isotopes ${ }^{27}$.

En janvier 1985, la Société de la recherche retient officiellement l'option d'un réacteur de type MAPLE, dont la puissance pourrait varier entre 10 et 20 mégawatts (d'où l'ajout d'un "X " à l'acronyme du réacteur pour MAPLE-X), qui serait construit dans le blindage du NRX ${ }^{28}$. Cependant, une fuite détectée au mois de mai dans un tube du NRU nécessite son arrêt pendant 5 mois et force la Société de la recherche à repenser sa stratégie. La construction du MAPLE-X à l'intérieur de l'enveloppe du NRX la prive en effet du réacteur de réserve nécessaire pour maintenir la continuité de sa production de radio-isotopes. Le MAPLE-X serait donc construit dans un nouveau bâtiment adjacent au NRX, de manière à ce que ce dernier puisse prendre le relai du NRU à tout moment ${ }^{29}$. Étant donné l'enjeu crucial que constitue la stabilité de la production des radio-isotopes pour la Société radiochimique, c'est elle qui devrait assumer les coûts additionnels de 1.9 millions de dollars $(\mathrm{M} \$)$ qu'aurait permis d'économiser une réutilisation partielle de l'infrastructure du NRX ${ }^{30}$.

\footnotetext{
26. Ibid., p. 4.

27. LCAI, A-2016-00027, "Presentation to the Board of Directors on MAPLE-X », 28 novembre 1985 , p. 6.

28. Heeds, juin 1985, p. 1.

29. LCAI, A-2016-00027, " Presentation to the AECL Board of Directors ", 25 janvier 1985, p. 6. 30. Ibid., p. 5.
} 
En novembre 1985, le projet de construction du MAPLE-X est approuvé par le C.A. d'ÉACL qui lui alloue un budget de 9.9 M\$. Sa finalité double de modèle de démonstration pour le marché des réacteurs de recherche et pour la production de radio-isotopes est de nouveau explicitée : "MAPLE-X would serve the dual role of demonstration reactor and primary supplier of radio-isotopes $^{31}$ ». La date de mise en service du réacteur est prévue pour le mois de juin 1988. Le président de la Société de la recherche, R.G. Hart, se montre très optimiste quant au bon déroulement du projet et entrevoit même la possibilité de pousser plus loin les capacités du réacteur une fois réalisées ses deux principales missions : "The reactor is quite simple and very reliable. Also MAPLE-X can be operated up to about $22 \mathrm{MW}$ with no modifications and up to $30 \mathrm{MW}$ with only slight modifications. It is therefore quite versatile to meet currently unforeseen needs ${ }^{32}$ ».

\section{Les contraintes techniques du projet MAPLE-X}

Avant de démarrer tout projet nucléaire, ÉACL doit obtenir une autorisation de la part de la CCEA, l'organisme de réglementation de l'industrie nucléaire au Canada. Les démarches nécessaires à l'obtention d'un permis sont menées par un groupe d'experts, le Nuclear Safety Advisory Committee (NSAC), lui-même chargé à l'interne d'ÉACL d'évaluer la sûreté du fonctionnement de ses propres installations. Le NSAC est composé d'ingénieurs provenant de différentes branches de l'entreprise, mais aussi d'observateurs issus de la CCEA. Il a pour tâche d'évaluer les analyses techniques de sûreté préparées par les ingénieurs d'ÉACL qui sont censées garantir le fonctionnement du réacteur MAPLE-X selon les normes établies par la CCEA. Le groupe fixe également les directives conduisant à la validation des différentes étapes d'obtention de la licence d'opération du réacteur.

Pour recevoir l'autorisation de passer d'une étape à l'autre, une série de rapports techniques soumis par ÉACL doivent être approuvés par la CCEA: d'abord, le Concept Safety Assessment Report, qui sollicite l'approbation de principe du concept du réacteur, le lieu de l'installation et l'autorisation de 
débuter certains travaux de chantiers; ensuite, le Preliminary Safety Analysis Report, qui sollicite l'obtention du permis de construction du réacteur; et enfin, le Final Safety Analysis Report qui sollicite l'autorisation de démarrer le réacteur et l'obtention du permis d'exploitation ${ }^{33}$. Bien qu'il obéisse à une logique cohérente, l'ordre de validation de chacune de ces étapes, et de la documentation technique qui leur est associée, n'est pas toujours respecté. Il peut en effet arriver qu'ÉACL demande l'autorisation de démarrer certains travaux de construction, alors que le concept n'a été approuvé que de façon partielle, si la CCEA juge que sa validation définitive n'aura pas d'impact sur les travaux déjà entrepris. Par exemple, les recherches menées pour fixer le choix du type de combustible du MAPLE-X10 sont toujours en cours au printemps $1986^{34}$, alors que les contrats pour le développement et la construction de la cuve du réacteur ont déjà été attribués aux compagnies Dominion-Bridge Sulzer et Titanium Limited ${ }^{35}$.

Alors que le démarrage du réacteur est prévu pour juin 1988, le projet commence à prendre du retard sur son calendrier dès l'été 1986. En effet, les premiers documents techniques n'ont pas obtenu l'aval préalable du NSAC, puisqu'une série de scénarios d'accidents devant être couvert par les études de sûreté n'a pas été prise en compte ${ }^{36}$. En mars 1987, à mesure que les détails de conception sont affinés, des augmentations de coût et des retards sont identifiés par la direction du projet: "As detailed design proceeded and code and licensing requirements became firmer, it was realized that some of the scope and significantly more licensing and code requirements were necessary ${ }^{37}$ ». Le coût de construction est déterminant pour la viabilité commerciale du MAPLE, puisqu'ÉACL cible le marché des pays émergents qui ne disposent pas de moyens financiers importants. Cette contrainte oblige la Société de la recherche à revoir les ambitions de son projet à la baisse, en proposant deux importantes modifications de conception au réacteur.

33. Cotnam, 1989, p. 121.

34. LCAI, A-2016-00027, "AECL Reserach Co. Business Opportunity Highlights », 31 mai 1986, p. 7.

35. Bibliothèque et Archives Canada (BAC), RG 19, BAN 2002-01191-5, PRI 3300-A0003-001, boîte 17, dossier 1V, 31 janvier 1986.

36. Ibid., p. 8-9.

37. LCAI, A-2016-00027, procès-verbal de la réunion du C.A. d'ÉACL, 27 novembre 1987, p. 15. 
D'abord, sa puissance de sortie maximale est limitée à $10 \mathrm{MW}$ au lieu des $20 \mathrm{MW}$ prévus initialement. Ce changement permettrait de réaliser des économies appréciables sur la construction du bâtiment enveloppant le réacteur, puisque la version à $20 \mathrm{MW}$ nécessite une structure qui est environ $50 \%$ plus volumineuse que celle d'un réacteur limité à $10 \mathrm{MW}$. Cette décision est loin de constituer un changement trivial, puisque le nouveau concept résultant de la diminution de la puissance du réacteur s'avère être: "very much different from that of the earlier MAPLE-X20 ${ }^{38}$ ". Un retour vers une puissance de $10 \mathrm{MW}$ signifie que la conception du réacteur, dont l'essentiel a été réalisé entre 1983 et 1986, doit être revisitée et faire l'objet d'une nouvelle validation par les membres du NSAC ${ }^{39}$.

La deuxième mesure prise par la direction du projet consiste à limiter les capacités de recherche du réacteur: «Now, the MAPLE will not have such capacities as neutron radiography (an imaging process) or the ability to extract neutron beams to test industrial components ${ }^{40}$ ". ÉACL va encore plus loin en janvier 1988 et annonce que le réacteur n'incorporera finalement plus aucune capacité de recherche et sera strictement dédié à la production de radio-isotopes ${ }^{41}$. La Société de la recherche vient en effet d'apprendre au C.A. d'ÉACL que les estimations des coûts du réacteur avoisinent désormais les $20 \mathrm{M} \$$, montant qu'elle ne peut défrayer étant donné son budget réduit. En échange d'un droit d'exclusivité sur sa future production de radio-isotopes, la Société radiochimique accepte, en avril 1988, d'assumer tous les coûts de construction du MAPLE-X10. Mais cela implique l'abandon de la dimension $R \& D$ du réacteur, que la Société radiochimique n'a aucun intérêt à financer dans le cadre de ses activités commerciales.

Le recentrage du projet MAPLE-X autour de la production des radio-isotopes ne signifie pas pour autant que son intérêt pour la direction d'ÉACL se limite uniquement à cette dimension ${ }^{42}$. En effet, l'intérêt du MAPLE-X10 réside surtout dans le fait qu'il permettra d'arrêter les réacteurs NRX et

38. LCAI, A-2015-00138, " MAPLE-X10 Schedule and Key Issues ", 17 novembre 1987, p. 1.

39. LCAI, A-2015-00138, « MAPE-X10 Key Issues », 19 novembre 1987, p. 1.

40. "AECL stops Maple's construction while it reviews reactor project ", The Globe and Mail, 23 juillet 1987, p. B14.

41. LCAI, A-2016-00027, procès-verbal de la réunion du C.A. d'ÉACL, 22 janvier 1988, p. 3.

42. LCAI, A-2016-00027, procès-verbal de la réunion du C.A. d'ÉACL (présentation de David Cuthbertson), 29 avril 1988. 
le WR-1 dans le cadre du programme de rationalisation des réacteurs de recherche, d'accroître la disponibilité du NRU pour la R\&D et de démontrer la faisabilité technique du concept MAPLE. ÉACL vient en effet de signer un contrat avec l'agence nucléaire sud-coréenne KAERI pour la conception d'un réacteur MAPLE de $30 \mathrm{MW}^{43}$, et des négociations sont en cours avec le gouvernement de la Colombie pour la livraison clés en main d'un MAPLE d'une puissance de $1 \mathrm{MW}$ qui comprenne des capacités de recherche et de production de radio-isotopes ${ }^{44}$.

En dépit de la diminution de la portée du projet MAPLE-X, la Société de la recherche prévoit encore une nouvelle augmentation de ses coûts en capital à 22.6 $\mathrm{M} \$$ et repousse la date officielle de mise en service du réacteur au 30 septembre $1991^{45}$. Des modifications continuent d'être apportées à des éléments fondamentaux du concept, tel que le combustible du réacteur qui passe d'une composition de dioxyde d'uranium $\left(\mathrm{UO}_{2}\right)$ enrichi à hauteur de 5 à $8 \%$, vers un alliage d'uranium, de silicium et d'aluminium $\left(\mathrm{U}_{3} \mathrm{Si}-\mathrm{Al}\right)$ enrichi à hauteur de $20 \%$, option qui optimise davantage les flux neutroniques pour la production des radio-isotopes ${ }^{46}$. Loin de l'optimisme affiché au départ du projet, la requête de financement additionnel soumise par la Société de la recherche au C.A. d'ÉACL précise désormais que le réacteur n'est qu'un prototype et que les estimations seront probablement encore appelées à augmenter ${ }^{47}$.

La principale raison du retard pris par le projet réside dans une divergence de vue entre les ingénieurs d'ÉACL et les agents de la CCEA sur la définition des critères de sûreté du MAPLE-X10. Du côté d'ÉACL, on considère que la puissance du réacteur, abaissée à $10 \mathrm{MW}$, devrait lui permettre de définir des critères moins contraignants que ceux qui s'appliquent aux centrales électronucléaires CANDU, dont la puissance beaucoup plus élevée varie entre 600 et $800 \mathrm{MW}$. Des critères de sûreté moins restrictifs impliquent une conception simplifiée et, par conséquent, moins coûteuse

43. Ray Silver, "KAERI to build research reactor combining two Canadian designs ", Nucleonics Week, vol. 29, no. 5, 4 février 1988, p. 5.

44. Ray Silver, «AECL working on four projects for MAPLE research reactors », Nucleonics Week, vol. 29 , no. 50,15 décembre 1988 , p. 8.

45. BAC, RG 19, PRI 3300-N0002, 2007-00309-0, boîte 2, dossier 1 PB, 3 novembre 1993.

46. "Briefly...Canada », Nucleonics Week, vol. 29, no. 25, 23 juin 1988, p. 14. Pour une comparaison entre les configurations de cœur utilisant $\mathrm{UO}_{2}$ et $\mathrm{U}_{3} \mathrm{Si}-\mathrm{Al}$, voir Smith, 1986.

47. BAC, RG 19, PRI 3300-N0002, 2007-00309-0, boîte 2, dossier 1 PB, 3 novembre 1993. 
des systèmes de sûreté du réacteur. L'argument est cependant loin d'être partagé par les agents de la CCEA. L'un d'entre eux, P.M. French, note le manque d'expérience de son équipe dans un projet tel que celui du MAPLE-X10, puisqu'aucun critère n'a encore été établi pour des réacteurs dans sa gamme de puissance. Cela est dû au fait qu'il n'y a pas eu de « petits réacteurs " construits au Canada depuis près de trente ans, tandis que la réglementation nucléaire a, de façon générale, considérablement évolué depuis $^{48}$.

La CCEA considère qu'une cohérence devrait être maintenue entre les réacteurs de type MAPLE et CANDU : " acceptance criteria proposed for MAPLE should not be inconsistent with those in effect for power reactors $^{49}$ ". En particulier, les doses de radioactivité maximales, pouvant être reçues par un individu ou une population en cas d'accident, ne devraient pas être supérieures à celles définies pour les réacteurs de haute puissance. Ceci dit, la pertinence d'une adéquation totale entre les critères de sûreté de deux types de réacteurs aux caractéristiques et aux usages très différents se pose aussi. Par exemple, les doses radioactives maximales utilisées comme référence dans les centrales CANDU, comme celle de Darlington en Ontario, s'appliquent uniquement à des personnes se trouvant à l'extérieur du site délimitant le périmètre de la centrale et ne concernent pas le personnel travaillant sur le site. Le réacteur MAPLE-X10 doit lui être construit sur le site des laboratoires de Chalk River, un emplacement isolé, comprenant une large zone d'exclusion, mais qui concentre en son sein une population d'employés d'environ 2000 personnes. Il est donc indispensable que les normes conçues pour le MAPLE prennent pour critère limitatif la population qui se trouve sur le site et non pas celle qui se situe dans ses environs.

La question de la cohérence des normes de sûreté entre réacteurs de haute et de faible puissance se pose au niveau de la conception des deux systèmes d'arrêt d'urgence du MAPLE-X10 $0^{50}$, mais également au niveau du concept de ventilation d'urgence. Bien que les agents de la CCEA concluent, en octobre 1988, que la conformité de ce dernier demeure conditionnelle au respect des limites de doses radioactives reçues dans certains scénarios

48. LCAI, A-2015-00139, "Discussion paper on key issues identified in Maple-X10 concept ", 29 janvier 1988, p. 1.

49. Ibid., p. 2.

50. Ibid., p. 6. 
d'accident ${ }^{51}$, ils autorisent ÉACL à passer à l'étape de l'approbation de la construction du réacteur, qui est divisée en quatre phases : les trois premières pour le bâtiment du réacteur et la quatrième pour le réacteur et les différents systèmes qui lui sont associés. Alors que la CCEA valide les trois premières phases à l'été 1989, elle exige de la part d'ÉACL pour la quatrième la soumission complète de la documentation du concept MAPLE-X et de ses analyses de sûreté ${ }^{52}$.

\section{Les contraintes commerciales et politiques du projet MAPLE-X}

En mai 1989, alors que $75 \%$ de la conception du réacteur est complétée et que $80 \%$ des estimations ont déjà été reçus de la part des fournisseurs d'équipement, il devient évident que le budget et le calendrier fixés au printemps 1988 ne seront pas respectés ${ }^{53}$. En août, les nouvelles estimations indiquent une augmentation des coûts en capital du projet à 36.6 M\$. La mise en service du réacteur est une nouvelle fois repoussée à mars 1992. Cette situation pousse le nouveau président de la Société de la recherche, Terry Rummery, à suspendre l'ensemble des travaux, le temps de réévaluer les options financières d'ÉACL sur un projet qui a pâti : " from inexperience in projecting costs and associated risks while it was at the conceptual stage ${ }^{54} »$.

En septembre 1989, trois options s'offrent au C.A. La première est de mettre un terme à la construction du réacteur MAPLE-X10 et de se retirer du marché des radio-isotopes ${ }^{55}$. La seconde option, moins radicale, consiste tout de même à mettre fin au projet, tout en maintenant la production des radio-isotopes dans le NRU. Cette option oblige néanmoins ÉACL à trouver des fournisseurs tiers pour la Société radiochimique sur le moyen terme, car le NRX ne pourra continuer à tenir sa fonction de réacteur de réserve que pour quelques années, étant donné son âge avancé. Finalement, la troisième option consiste à poursuivre le projet, malgré l'escalade des coûts. Le C.A. choisit cette dernière option, car les deux

51. LCAI, A-2015-00138, BMD 93-160, 14 septembre 1993, p. 2.

52. Ibid.

53. LCAI, A-2016-00027, procès-verbal de la réunion du C.A. d'ÉACL, 29 septembre 1989, p. 10.

54. Ibid., p. 10.

55. Ibid., p. 11. 
premières occasionneraient d'importantes pénalités dues à des ruptures de contrat commerciaux avec les clients de la Société radiochimique. De plus, les revenus issus de la production des radio-isotopes ont pris une importance considérable dans le bilan comptable d'ÉACL, au contraire de la division d'ingénierie du CANDU qui traverse une période difficile en l'absence de nouvelles commandes de centrales électronucléaires. Ainsi, pour l'année 1989, ÉACL prévoit que la Société radiochimique devrait réaliser 23.6 $\mathrm{M} \$$ en revenus net, tandis la division CANDU devrait essuyer un déficit de $3 \mathrm{M} \${ }^{56}$.

Au début de l'année 1990, deux des principaux réacteurs de production de radio-isotopes dans le monde, DIDO en Angleterre et CINTICHEM aux États-Unis, doivent subitement être fermés, après que des failles eurent été identifiées dans leur fonctionnement. Le volume de production du NRU connait alors une croissance mécanique de $40 \%$ par rapport à son niveau de $1988^{57}$, en particulier pour des radio-isotopes d'importance majeure pour la médecine nucléaire tels que le molybdène-99, l'iode-125 ou l'iode131. La Société radiochimique se voit propulsée dans une situation quasi-monopolistique, avec des parts de marché pouvant atteindre $90 \%$ sur certains radio-isotopes. Globalement, elle assume $65 \%$ de la production mondiale des radio-isotopes médicaux, avec des revenus qui augmentent en moyenne de $16 \%$ par année depuis 1980, passant de 12 à $45 \mathrm{M} \$$ en 1989. Sa division d'irradiation industrielle, basée sur la commercialisation du cobalt-60, voit quant à elle ses revenus croître en moyenne de $19 \%$ par année dans la même période, passant de 14 à $68 \mathrm{M} \$ \$^{58}$.

Cependant, du point de vue de la Société de la recherche, l'augmentation du rythme de la production de radio-isotopes dans le NRU est problématique car, en plus de contrarier ses activités de $R \& D$, il en résulte une quantité de déchets radioactifs à traiter bien plus élevée que ce à quoi ses installations sont habituées ${ }^{59}$. La question du coût de la gestion des déchets radioactifs devient alors majeure dans le cadre du projet MAPLE-X10,

56. BAC, RG 19, vol. 6780, dossier 7 PA, «AECL Situation Report », hiver 1986-87, p. 2.

57. BAC, RG 19, PRI 3300-N0002, 2007-00309-0, boîte 2, dossier 1 PB, " The AECL Radioisotope Production and Service Operation ", janvier 1991, p. 19.

58. Rapport annuel ÉACL 1989-90, p. 18; BAC, RG 19, BAN 2007-00309-0, PRI 3300-N0002, boîte 2, dossier 1 PB, "Privatisation de Nordion International Inc. », novembre 1990, p. 9.

59. LCAI, A-2016-00027, "The AECL Radioisotope Production Operation over the Next Decade ", 30 novembre 1990 , p. $2-3$. 
puisque l'ajout de nouvelles installations s'avère nécessaire, si la Société de la recherche veut être en mesure de traiter l'ensemble des déchets qui seront produits par son futur réacteur.

La contrainte exercée sur le projet MAPLE-X par la croissance de la demande mondiale en radio-isotopes est accentuée par la volonté du gouvernement canadien de privatiser la Société radiochimique ${ }^{60}$. Deux organismes gouvernementaux pilotent cette opération depuis février 1987, le Bureau de la privatisation et des affaires réglementaires et la Corporation de développement des investissements du Canada (CDIC), qui redéfinit à l'automne 1988 les ententes commerciales liant la Société radiochimique et la Société de la recherche. Ces ententes font l'objet de sévères critiques de la part d'ÉACL qui reproche notamment à la CDIC d'en avoir fait des instruments pour augmenter la valeur à la vente de la Société radiochimique, au détriment des intérêts stratégiques de la Société de la recherche ${ }^{61}$.

Deux termes en particulier remettent directement en cause la pérennité de son activité de producteur de radio-isotopes et, par conséquent, la viabilité du projet MAPLE-X10. Premièrement, la CDIC a déterminé la part des revenus de la Société de la Recherche sur la commercialisation des radio-isotopes qu'elle produit par un pourcentage fixe des profits nets réalisés par la Société radiochimique sur ses ventes. Les termes de ce partage ne tiennent pas compte de l'augmentation des coûts de production, dus notamment à l'augmentation du volume des déchets radioactifs, ni de l'efficacité opérationnelle de la future société privatisée. Autrement dit, du point de vue d'ÉACL, il n'existe plus de relation directe entre les coûts assumés par la Société de la recherche et les revenus qu'elle perçoit. Deuxièmement, le contrat d'approvisionnement de 23 ans sensé lier la Société de la recherche à la future société privatisée ne comporte aucune provision de réouverture ou d'ajustement à l'augmentation des coûts de production. ÉACL affirme qu'elle a accepté ces termes car elle pensait recevoir en contrepartie l'intégralité des revenus de la privatisation ${ }^{62}$, mais le gouvernement, sous l'influence du Ministère des finances, décide finalement que cette rentrée d'argent servira plutôt à combler le déficit public.

60. Khelfaoui, 2020.

61. Ibid., p. 1.

62. BAC, RG 19, BAN 2014-00484-6, 3300-N0002, boîte 1, dossier 2V, $1^{\text {er }}$ mars 1991. 
L'augmentation de la cadence de production des radio-isotopes fait peser sur le NRU, un réacteur âgé de 34 ans, une pression qui devient difficile à soutenir. En janvier 1991, ÉACL se voit contrainte de mettre son réacteur à l'arrêt pour une durée indéterminée, à la suite d'un bris dans une conduite de son système de refroidissement. Cet incident force la Société de la recherche à y effectuer des réparations durant toute l'année 1991, tandis que le NRX prend le relai de la production. Entre temps, la construction du bâtiment du réacteur MAPLE-X10 est achevée au printemps 1991. Les commissaires de la CCEA autorisent même l'installation de certains systèmes et équipements, même si ses agents notent que le rythme de la construction devance trop celui de la production des analyses de conception et de sûreté du réacteur, une situation risquant d'aboutir à une "installation insatisfaisante ${ }^{63}$ ". De fait, au mois de juillet, ÉACL informe la CCEA que l'utilisation d'un programme informatique permettant une modélisation plus fine de certains phénomènes thermo-hydrauliques indique la violation d'un critère de sûreté du réacteur pour certains scénarios d'accident ${ }^{64}$. La date de mise en service du réacteur est une fois de plus reportée au mois d'octobre 1994.

En novembre 1991, l'entreprise pharmaceutique privée MDS Health acquiert la Société radiochimique de la part du gouvernement du Canada pour un montant de $165 \mathrm{M} \$$ et la renomme MDS Nordion. Dans une lettre adressée à son homologue de la CDIC, le président de MDS Health, W.G. Lewitt, s'inquiète aussitôt de l'allongement des délais de remise en service du NRU et de la dépendance de la Société radiochimique au seul réacteur NRX qui en résulte, en attendant la mise en service du MAPLE$\mathrm{X} 10^{65}$. Ses préoccupations sont d'autant plus légitimes qu'après avoir fonctionné en relai du NRU durant 42 semaines consécutives, c'est au tour du NRX d'être soumis à un arrêt forcé pour des réparations d'urgence, en janvier 1992. Le réacteur de soutien, âgé de 44 ans, n'a probablement pas soutenu la cadence d'une opération à laquelle il n'avait plus été soumis depuis $1986^{66}$. Les travaux de réparations du NRX se poursuivent durant

63. BAC, RG 60, accès 1993-94/093, BMD 91-179/91-201, boîte 31, BMD 91-196, 7 novembre 1991, p. 4.

64. LCAI, A-2015-00138, BMD 92-210A, 10 décembre 1992, p. 2.

65. BAC, RG 19, BAN 2014-00484-6, 3300-N0002, boîte 1, dossier 2V, 23 août 1991.

66. BAC, RG 60, BMD 91-198, BMD 91-179/91-201, accès 1993-94/093, boîte 31, 22 octobre 1991, p. 1. 
un an et sont estimés à 29.5 M\$ en janvier 1993, sans que les ingénieurs d'ÉACL ne puisse en garantir le fonctionnement sécuritaire ${ }^{67}$.

Au début de l'année 1993, le C.A. d'ÉACL décrit l'implication de l'entreprise dans la production des radio-isotopes pour le compte de MDS Nordion comme: "the largest issue facing the Corporation ${ }^{68}$ ". À court et à moyen terme, des fonds sont nécessaires pour réaliser trois opérations coûteuses et aux issues incertaines : la mise en service du réacteur MAPLE-X10, la construction de nouvelles installations de gestion et de stockage des déchets radioactifs et le rétablissement du réacteur NRX. Ces coûts vont bien au-delà des capacités financières d'ÉACL, d'autant plus que cette dernière souffre d'un important manque de liquidités depuis la privatisation de la Société radiochimique. La Société de la recherche se voit contrainte d'appliquer un contrôle strict de ses dépenses et même, dans certains cas, de procéder à des réductions d'effectifs : "Staff and other cost reduction action is being taken by AECL Research, principally in $R \& D$ support areas, to lessen the impact of the isotope cash drain ${ }^{69}$ ".

Dans ce contexte, le C.A. d'ÉACL estime que la poursuite de son activité sur le marché mondial des radio-isotopes sera déficitaire ${ }^{70}$, d'autant plus que le vice-président aux opérations de la Société de la recherche, Mike Wright, confirme au C.A. qu'il ne peut garantir que l'exploitation du NRX pourra se faire dans le respect des normes de sécurité établies par la $\mathrm{CCE}^{71}$. Le 29 mars, la direction d'ÉACL avertit MDS Nordion que "le réacteur NRX, utilisé depuis plus de 46 ans, sera fermé en permanence parce qu'[elle] ne peut plus garantir la sécurité de son exploitation $^{72}$ ». Quelques semaines plus tard, le président de la Société de la recherche, Terry Rummery, confirme au C.A. d'ÉACL que l'achèvement de la construction du MAPLE-X10 comporte un risque financier considérable : " further design changes to meet licensing requirements could drive up the cost and increase the projected timing to completion ${ }^{73}$ ". Les coûts en capitaux du projet ont augmenté d'environ $65 \mathrm{M} \$$ depuis la pri-

67. LCAI, procès-verbal de la réunion du C.A. d'ÉACL, 22 janvier 1993, p. 9.

68. Ibid p. 5.

69. Ibid. p. 6.

70. LCAI, A-2016-00027, procès-verbal de la réunion du C.A. d'ÉACL, 26 février 1993, p. 5.

71. Ibid

72. BAC, RG 19, 2014-00484-6, 3300-N0002, boîte 1, dossier 2V, 16 juillet 1993, p. 2.

73. LCAI, A-2016-00027, procès-verbal de la réunion du C.A. d'ÉACL, 23 avril 1993, p. 8. 
vatisation de la Société radiochimique et sont à présent estimés à $105 \mathrm{M} \$$, si l'on prend en compte le coûts des nouvelles installations de gestion des déchets radioactifs.

En mai 1993, le président d'ÉACL, Bruce Howe, avertit son vis-à-vis de MDS Health que son entreprise ne peut continuer à dépenser des fonds dans le projet MAPLE-X10, ajoutant qu'ÉACL n'a aucune obligation contractuelle de poursuivre le projet ${ }^{74}$. Le 25 mai, ÉACL informe officiellement MDS Health que, dans l'éventualité où elle ne pourrait " recouvrer ses coûts et générer un rendement commercial acceptable, elle n'aurait d'autres alternatives que de limiter au minimum ses activités dans la production des radio-isotopes " et, éventuellement, "se retirer complètement de ce secteur d'activitét ${ }^{\prime \prime}$. Cela conduit le C.A. d'ÉACL à suspendre un certain nombre d'opérations en cours sur le projet MAPLE-X10 jusqu'en septembre, moment où une décision définitive concernant le projet devrait être prise $^{76}$.

\section{Le MAPLE-X devant la CCEA: approbation du concept, abandon du projet}

Au mois de septembre 1993, le MAPLE-X10 traverse une étape cruciale de sa validation conceptuelle : la CCEA doit se prononcer sur la demande d'obtention du permis de construction du réacteur. Trois mois auparavant, ÉACL avait soumis une demande d'" approbation hâtive ", prétextant la nécessité de respecter ses obligations contractuelles envers MDS Nordion. Les cinq commissaires de la CCEA jugent qu'une revue détaillée des analyses de sûreté du MAPLE-X10 prendra du temps et qu'elle sera difficile à réaliser à l'intérieur du délai demandé. Cependant, ils se disent prêt à accorder une approbation conditionnelle, si ÉACL est en mesure de proposer une solution satisfaisante aux plus importants problèmes de conception et de sûreté qui ont déjà été soulevés par ses agents, sans que cela n’ait d'impact sur les systèmes du réacteur qui ont déjà été installés ${ }^{77}$.

\footnotetext{
74. BAC, RG 19, 2014-00484-6, 3300-N0002, boîte 1, dossier 2V, 2 septembre 1993, p. 1. 75. Ibid.

76. LCAI, A-2016-00027, procès-verbal de la réunion du C.A. d'ÉACL, 18 juin 1993, p. 6. 77. LCAI, A-2016-00027, "AECB Internal Meeting on MX10 Safety Analysis », 24 juin 1993, p. 1 .
} 
Le 14 septembre, la direction de la réglementation des réacteurs et celle de l'analyse et de l'évaluation présentent aux commissaires de la CCEA leurs recommandations. Un premier grief important concerne les écarts de méthodologie qui ont pu être constatés entre l'approche utilisée dans les projets des centrales CANDU et celui du MAPLE : «staff reviewed the methodology proposed by AECL and identified several significant issues. These centered on the question of whether power reactor safety analysis rules were applicable to a low power reactor like $\mathrm{MAPLE}^{78}$ ". Une revue des accidents de référence réalisée par les agents de la CCEA laisse également planer un doute sur l'efficacité du premier système d'arrêt d'urgence du réacteur. Étant donné l'usage extensif prévu du MAPLE-X10 pour la production des radio-isotopes, sa conception requiert de prendre en compte des changements de combustible fréquents, pouvant atteindre un rythme hebdomadaire. Une erreur de rechargement pourrait risquer d'endommager le cour du réacteur. Dans certains cas de figure liés à cet évènement, l'efficacité du premier système d'arrêt d'urgence est remise en question. Une manière de contourner le problème consisterait à démontrer que l'évènement a une probabilité si faible de survenir qu'il pourrait être exclu de la liste des accidents à couvrir par les systèmes de sûreté du réacteur. Mais pour cela, la CCEA exige qu'ÉACL lui fournisse une preuve nette que les conséquences de l'évènement ne mènent pas à des situations jugées inacceptables du point de vue de la sûreté nucléaire.

Les agents de la CCEA portent également une attention particulière à la conception du bâtiment du réacteur. Ils s'interrogent sur sa robustesse et sur sa capacité à confiner les rejets radioactifs découlant d'un accident qui impliquerait le blocage du débit d'un canal du réacteur. Le concept de ventilation d'urgence du bâtiment a aussi été examiné, à travers le calcul des doses prédites aux employés des laboratoires de Chalk River et aux habitants des lieux résidentiels environnants. Si la CCEA ne considère pas les doses calculées par ÉACL comme "inacceptables ", elle relève néanmoins la présence d'importantes incertitudes dans les hypothèses utilisées pour calculer l'étendue des dommages au combustible, le volume et la composition isotopique des rejets radioactifs ainsi que leur dispersion dans l'atmosphère. La prise en compte et l'addition de marges d'erreur plus élevées pourrait mener à une réévaluation à la hausse de la quantité de

78. LCAI, A-2015-00138, BMD 93-160, 14 septembre 1993, p. 6. 
doses rejetée au public, ce qui nécessiterait de revoir une nouvelle fois la conception du bâtiment ${ }^{79}$.

Pour conclure, les agents de la CCEA estiment que les questions relatives au premier système d'arrêt d'urgence et à la conception du bâtiment du réacteur doivent être réglées avant qu'ÉACL ne puisse recevoir l'approbation du permis de construction. Leur jugement est renforcé par le fait qu'ÉACL leur a soumis une quantité importante de documents techniques durant les derniers mois qui n'ont pas encore été examinés. Des documents supplémentaires sont encore attendus par la CCEA et des discussions et des rencontres techniques continuent à se tenir entre les deux parties.

Cependant, à peine un mois après avoir rendu un premier avis défavorable, et malgré l'ampleur du travail qui reste à accomplir, les agents de la CCEA décident de renverser leur jugement et recommandent finalement l'approbation de la construction du réacteur ${ }^{80}$. En préambule de leur décision, ils rappellent pourtant que le document qu'ils avaient soumis aux commissaires en septembre "fait état de plusieurs problèmes qui auraient pu avoir des répercussions au plan technique et qui devraient être réglés avant qu'une recommandation ne puisse être faite, notamment : la marge de réactivité du système de sûreté numéro un, et la conception du bâtiment réacteur $^{81}$ ". ÉACL s'étant entre temps engagée à apporter des modifications techniques et opérationnelles pour corriger ces deux situations, les agents sont à présent d'avis que les mesures proposées « sont acceptables en principe et que les problèmes relevés ne constituent plus un empêchement à l'approbation de construire ${ }^{82} "$.

Il est difficile d'établir avec certitude dans quelle mesure le conflit commercial larvé entre ÉACL et MDS Nordion, ainsi que la fermeture définitive du NRX ont pu influencer la recommandation des agents la CCEA. Ces derniers ont néanmoins bien pris en considération dans leur mémoire qu'ÉACL demandait " une approbation hâtive afin de respecter ses échéanciers d'approvisionnement de radio-isotopes à des fins médicales [...] cet approvisionnement [étant] à l'heure actuelle assuré uniquement par le

79. Ibid., p. 7.

80. Document CCEA, " Permis de construire la phase 4 du réacteur Maple-X10 aux Laboratoires de Chalk River ", BMD 93-188, LCAI, 19 octobre 1993.

81. Ibid., p. 1.

82. Ibid. 
réacteur $\mathrm{NRU}^{83}$ ". De son côté, le C.A. d'ÉACL interprète la recommandation favorable de la CCEA comme une approbation réglementaire de la technologie MAPLE qui devrait faciliter sa vente à l'étranger. Néanmoins, puisque rien dans son entente commerciale avec MDS Nordion ne l'oblige à construire effectivement le MAPLE-X10 qui s'avère déjà un gouffre financier, il n'y a désormais pour ÉACL plus aucune raison de poursuivre le projet, choix qui est validé par une décision de son C.A. le 29 octobre $1993^{84}$. Labandon du projet MAPLE-X ne marque pas pour autant la fin de l'activité de production de radio-isotopes médicaux pour la Société de la recherche, puisque celle-ci se poursuivra dans le réacteur NRU jusqu'à son arrêt définitif en $2016^{85}$.

\section{Les temporalités du projet MAPLE-X}

L'histoire du réacteur MAPLE-X montre que les trajectoires des GPT ne devraient pas être analysées comme une succession logique d'étapes menant à la réussite ou à l'échec de la réalisation d'une infrastructure, mais plutôt comme un processus « dynamique, souvent chaotique, de mise en convergence d'informations, d'adaptation à des contraintes et d'arbitrage entre des voies de développement divergentes ${ }^{86} "$. Entre le moment où il est approuvé en 1985 et celui où il est abandonné en 1993, le projet MAPLE-X doit en effet se plier à des contraintes issues d'adaptations techniques, d'exigences réglementaires, d'évolutions de contextes commerciaux et de décisions politiques. La trajectoire du réacteur MAPLE-X10 se dessine ainsi au carrefour des tensions qui se créent entre la tradition technologique d'ÉACL incarnée par les intérêts de la Société de la recherche, sa mission commerciale incarnée par son activité de producteur de radio-isotopes, sa dépendance aux politiques gouvernementales qui s'exprime dans la privatisation de la Société radiochimique, et les contraintes normatives qui s'appliquent au projet à travers les exigences de la CCEA.

Loin d'être linéaire, la trajectoire du projet MAPLE-X laisse entrevoir le déroulement de plusieurs temporalités qui s'enchevêtrent et s'influencent mutuellement. La temporalité technique joue un rôle majeur dans les premières

83. LCAI, BMD 93-160, op. cit., p. 1.

84. LCAI, A-2016-00027, procès-verbal de la réunion du C.A. d'ÉACL, 29 octobre 1993, p. 5.

85. Khelfaoui, 2019.

86. Lascoumes, 2004, p. 6. 
années du projet où des choix cruciaux sont fixés tels que la puissance de sortie du réacteur, le type de combustible qui y sera incorporé ou celui de ses systèmes de sûreté. Les changements successifs apportés aux caractéristiques techniques du MAPLE-X sont néanmoins grandement influencés par l'évolution de la situation financière de la Société de la recherche ainsi que la nouvelle position dominante de la Société radiochimique sur le marché mondial des radio-isotopes. La temporalité gestionnaire, dont relèvent ces deux derniers facteurs, est ainsi à l'origine de décisions techniques et de points de bifurcation majeurs dans le projet, telle que la limitation de la puissance de sortie du réacteur de 20 à $10 \mathrm{MW}$, l'abandon de ses capacités expérimentales ou la nécessité de lui adjoindre de nouvelles installations de gestion des déchets radioactifs. L'influence de la temporalité gestionnaire sur le projet s'exprime également à travers la mise en œuvre du Research Reactor Rationalization Program par la Société de la recherche, programme qui définit la finalité double, commerciale et expérimentale, du réacteur.

La définition des critères de sûreté du réacteur MAPLE-X10 est également fortement tributaire de contraintes réglementaires. Les échanges réguliers entre ingénieurs d'ÉACL et agents de la CCEA par rapport à cette question contribuent grandement à l'incertitude qui caractérise le projet. Ils montrent également que la temporalité réglementaire ne consiste pas simplement à évaluer, à des moments précis du projet, la conformité du réacteur à l'aune de normes techniques rigides. Cette temporalité s'apparente plutôt à un processus faisant converger la conception du réacteur vers une forme négociée, qui prend non seulement en compte les exigences de la réglementation, mais considère également les contraintes extra-techniques pesant sur le projet. Ainsi, la décision finale de la CCEA d'octroyer le permis de construction du MAPLE-X10 considère clairement la nécessité pour ÉACL : "de respecter ses échéanciers d'approvisionnement de radio-isotopes à des fins médicales ${ }^{87}$ ".

La temporalité politique agit d'abord sur le projet MAPLE-X à travers la décision gouvernementale de réduire le budget de la Société de la recherche, qui conduit cette dernière à penser l'exploitation de son futur réacteur dans le cadre plus large d'un programme de rationalisation de ses activités de recherche. Mais la temporalité politique pèse surtout à travers la décision du gouvernement de privatiser la Société radiochimique,

87. LCAI, BMD 93-160, op. cit., p. 1. 
privatisation qui finit par remettre en cause la rentabilité économique du projet et conduit ultimement ÉACL à l'abandonner. Réacteur expérimental devant consacrer la validité conceptuelle d'une technologie tout autant que réacteur commercial, le réacteur MAPLE-X10 ne cesse de porter en lui des finalités multiples tout au long du projet. Celles-ci reflètent les intérêts et les visions des différentes parties prenantes du projet (ingénieurs, managers, régulateurs, gouvernants), qui ne sont jamais mieux exposés qu’à travers l'analyse des différents espaces (technique, commercial, réglementaire, politique) où se trame l'histoire du projet MAPLE-X, justifiant ainsi son analyse sous l'angle des temporalités.

\section{Sources}

Bibliothèque et archives Canada, Fonds du ministère des Finances (RG 19).

Bibliothèque et archives Canada, Fonds CCEA/CCSN (RG 60).

Loi canadienne sur l'accès à l'information (requêtes A-2015-00138 et A-2016-00027).

"AECL stops Maple's construction while it reviews reactor project », The Globe and Mail, 23 juillet 1987, p. B14.

Agence Internationale de l'Énergie Atomique, Manpower Development for Nuclear Power. A Guidebook, STI/DOC/10/200, 1980.

"Briefly...Canada », Nucleonics Week, vol. 29, no. 25, 23 juin 1988, p. 14.

Cotnam K.D., "Safety Assessment of the MAPLE-X10 Reactor ", Proceedings of the International Symposium on Research Reactor Safety, Operations and Modifications, Rapport ÉACL-9926/ IAEA-SM-310, 23-27 octobre 1989.

Heeds W., "The MAPLE-X Concept Dedicated to the Production of Radioisotopes ", Rapport AECL-8837, juin 1985. https://inis.iaea.org/collection/ NCLCollectionStore/_Public/17/062/17062899.pdf?r=1

Lidstone R.F., « MAPLE: Un concept de réacteur canadien à usage multiple pour le développement nucléaire national ", Rapport ÉACL-7826, juin 1984.

Rapport annuel ÉACL 1989-90. https://inis.iaea.org/collection/ NCLCollectionStore/_Public/23/079/23079869.pdf

SiLVER Ray, "KAERI to build research reactor combining two Canadian designs ", Nucleonics Week, vol. 29, no. 5, 4 février 1988, p. 5.

SILVER Ray, "AECL working on four projects for MAPLE research reactors ", Nucleonics Week, vol. 29, no. 50, 15 décembre 1988, p. 8.

Sмiтн H.J. et al., "Selecting a MAPLE Research Reactor for 1-10 MW Operation ", rapport ÉACL-9060, juin 1986. 


\section{Bibliographie}

Barthe Yannick, Le pouvoir d'indécision. La mise en politique des déchets nucléaires, Économica, Paris, 2006.

Bijker Wiebe, Hughes Thomas P. et Pinch Trevor (dir.), The Social Construction of Technological Systems. New Directions in the Sociology and History of Technology, MIT Press, Cambridge, 1987.

Bothwell Robert, Nucléus. Histoire de l'Énergie atomique du Canada limitée, Édition de l'Arc, Montréal, 1988.

Bromberg Joan Lisa, "TFTR: The Anatomy of a Programme Decision », Social Studies of Science, $\mathrm{n}^{\circ} 4,1982$, p. 559-583.

BRUnET Philippe, «Temporalités dans la recherche biomédicale : la science au travail saisie par le temps ", La nouvelle revue du travail, $\mathrm{n}^{\circ}$ 1, 2012, p. 1-19.

Brunet Philippe et Dubors Michel, "Cellules souches et technoscience : sociologie de l'émergence et de la régulation d'un domaine de recherche biomédicale en France ", Revue française de sociologie, n 3, 2012, p. 391-428.

Cowan Robin, " Nuclear Power Reactors: A Study in Technological Lock-in ", Journal of Economic History, n 3, 1990, p. 541-567.

Dubar Claude, "Temporalité, temporalités : Philosophie et sciences sociales ", Temporalités, $\mathrm{n}^{\circ}$ 8, 2008. https://journals.openedition.org/temporalites/137.

Faucher Philippe (dir.), Grands projets et innovations technologiques au Canada, Presses de l'Université de Montréal, Montréal, 1999.

Gingras Yves et Khelfaour Mahdi, "La centrale nucléaire Gentilly-1 : La trajectoire imprévisible d'une innovation technologique avortée ", Revue d'histoire de l'Amérique française, $\mathrm{n}^{\circ}$ 1, 2013, p. 57-81.

Gingras Yves et Trépanier Michel, "Le Tokamak de Varennes et le programme canadien de fusion nucléaire : anatomie d'une décision ", Recherches Sociographiques, $\mathrm{n}^{\circ}$ 3, 1989, p. 421-446.

Giraudeau Martin et Graber Frédéric, «Le seuil de l'action. La décision préalable dans l'histoire des projets ", Entreprises et histoire, n 4, 2019, p. 40-57.

Graber Frédéric, "Du faiseur de projet au projet régulier dans les Travaux Publics (XVIII ${ }^{\mathrm{e}}$-XIX ${ }^{\mathrm{e}}$ siècles) : pour une histoire des projets ", Revue d'histoire moderne \& contemporaine, $\mathrm{n}^{\circ} 3,2011$, p. 7-33.

Grubler Arnulf, "The Costs of the French Nuclear Scale-Up: A Case of Negative Learning by Doing ", Energy Policy, n 9, 2010, p. 5174-5188.

Неснт Gabrielle, Le rayonnement de la France. Énergie nucléaire et identité nationale après la seconde guerre mondiale, La Découverte, Paris, 2014.

Hurst Donald G. (dir.), Canada Enters the Nuclear Age. A Technical History of 
Atomic Energy of Canada as Seen from its Research Laboratories, McGill-Queen's University Press, Montreal, 1997.

Jobert Arthur et Le Renard Claire, "Framing Prototypes: The Fast Reactor Breeder in France (1950s-1960s) », Science \& Technology Studies, vol. 27, n 2, 2014, p. 7-26.

Khelfaoui Mahdi, "Selling of the Crown Jewels: Socialization of Costs and Privatization of Profits in the Canadian Isotopes Industry ", Canadian Historical Review, vol. 101, n², 2020, p. 217-240.

KHELFAOUI Mahdi, La trajectoire sociohistorique d'un grand projet nucléaire : Les réacteurs MAPLE et l'industrie canadienne des isotopes médicaux, Thèse de doctorat, Université du Québec à Montréal, 2019.

Lascoumes Pierre et Le Galès Patrick, Sociologie de l'action publique, Armand Colin, Paris, 2007

Lascoumes Pierre, "La Gouvernementalité : de la critique de l'État aux technologies du pouvoir ", Le Portique, vol. 13-14, 2004.

MANGEON Michael, Conception et évolution du régime français de régulation de la sûreté nucléaire (1945-2017) à la lumière de ses instruments : une approche par le travail de régulation, Thèse de doctorat, Mines ParisTech, 2018.

Mangeon Michaël et Pallez Frédérique, « Réguler les risques nucléaires par la souplesse : genèse d'une singularité française (1960-1985) ", Annales des Mines Gérer et comprendre, $\mathrm{n}^{\circ} 4,2017$, p. 76-87

Mccurdy Howard, The Space Station Decision: Incremental Politics and Technological Choice, Johns Hopkins University Press, Baltimore, 2007.

Montel Nathalie, Le chantier du canal de Suez (1859-1869). Une histoire des pratiques techniques, Presses de l'École Nationale des Ponts et Chaussées, Paris, 1998.

Pestre Dominique et JacQ François, «Une recomposition de la recherche académique et industrielle en France dans l'après-guerre, 1945-1970 : Nouvelles pratiques, formes d'organisation et conceptions politiques ", Sociologie du Travail, vol. 38, n³, 1996, p. 263-277.

Pestre Dominique, "The Decision-Making Processes for the Main Particle Accelerators Built Throughout the World from the 1930s to the 1970s ", History and Technology, $\mathrm{n}^{\circ} 1,1992$, p. 163-174.

Pinch Trevor et Bijker Wiebe, "The Social Construction of Facts and Artefacts: Or how the Sociology of Science and the Sociology of Technology Might Benefit Each Other ", Social Studies of Science, n 3, 1984, p. 399-341.

Schultz Émilien, «Le temps d'un projet. Les temporalités de financement sur projet dans un laboratoire de biophysique ", Temporalités, $\mathrm{n}^{\circ} 18,2013$. https:// journals.openedition.org/temporalites/2563 
Tillemant Stéphanie, Garcias Frédéric, Minguet Guy et Charue Duboc Florence, «Disentangling Exploitation and Exploration in Hybrid Projects: The Case of a New Reactor Development ", Project Management Journal, n 5, 2019, p. $538-553$

Topçu Sezin, La France nucléaire. L'art de gouverner une technologie contestée, Seuil, Paris, 2013.

Velho Léa et Pessoa Osvaldo, "The Decision-Making Process in the Construction of the Synchrotron Light National Laboratory in Brazil ", Social Studies of Science, $\mathrm{n}^{\circ} 2,1998$, p. 195-219.

WeLlock Thomas, "A Figure of Merit. Quantifiying the Probability of a Nuclear Reactor Accident ", Technology and Culture, vol. 58, n 3, 2017, p. 678-721.

\section{Lauteur}

Mahdi Khelfaoui est professeur au département des sciences humaines de l'Université du Québec à Trois-Rivières. Ses recherches portent notamment sur l'histoire de l'industrie nucléaire canadienne ainsi que sur la transformation des relations entre l'État canadien et ses entreprises technologiques depuis le début des années 1980. Ses plus récents articles ont été publiés dans Canadian Historical Review, Revue d'études canadiennes et Recherches sociographiques. Contact : mahdi.khelfaoui@uqtr.ca 